\title{
Computational storage: an efficient and scalable platform for big data and HPC applications
}

\author{
Mahdi Torabzadehkashi ${ }^{1,2^{*}}$ (D), Siavash Rezaei ${ }^{1,2}$, Ali HeydariGorji ${ }^{1,2}$, Hosein Bobarshad $^{2}$, Vladimir Alves ${ }^{2}$ \\ and Nader Bagherzadeh ${ }^{1}$
}

*Correspondence: torabzam@uci.edu

${ }^{1}$ University of California, Irvine (UCI), Irvine 92697, USA Full list of author information is available at the end of the article

\begin{abstract}
In the era of big data applications, the demand for more sophisticated data centers and high-performance data processing mechanisms is increasing drastically. Data are originally stored in storage systems. To process data, application servers need to fetch them from storage devices, which imposes the cost of moving data to the system. This cost has a direct relation with the distance of processing engines from the data. This is the key motivation for the emergence of distributed processing platforms such as Hadoop, which move process closer to data. Computational storage devices (CSDs) push the "move process to data" paradigm to its ultimate boundaries by deploying embedded processing engines inside storage devices to process data. In this paper, we introduce Catalina, an efficient and flexible computational storage platform, that provides a seamless environment to process data in-place. Catalina is the first CSD equipped with a dedicated application processor running a full-fledged operating system that provides filesystem-level data access for the applications. Thus, a vast spectrum of applications can be ported for running on Catalina CSDs. Due to these unique features, to the best of our knowledge, Catalina CSD is the only in-storage processing platform that can be seamlessly deployed in clusters to run distributed applications such as Hadoop MapReduce and HPC applications in-place without any modifications on the underlying distributed processing framework. For the proof of concept, we build a fully functional Catalina prototype and a CSD-equipped platform using 16 Catalina CSDs to run Intel HiBench Hadoop and HPC benchmarks to investigate the benefits of deploying Catalina CSDs in the distributed processing environments. The experimental results show up to $2.2 \times$ improvement in performance and $4.3 \times$ reduction in energy consumption, respectively, for running Hadoop MapReduce benchmarks. Additionally, thanks to the Neon SIMD engines, the performance and energy efficiency of DFT algorithms are improved up to $5.4 \times$ and $8.9 x$, respectively.
\end{abstract}

Keywords: Computational storage, In-storage processing, Near-data processing, SSD, Big data, Hadoop, HPC, DFT, System-on-chip 


\section{Introduction}

The modern human's life has been technologized, and nowadays, people rely on big data applications to receive services such as healthcare, entertainment, government services, and transportation in their day-to-day lives. As the usage of these services becomes universal, people generate more unprocessed data, which increases the demand for more sophisticated data centers and big data applications. At current pace, 2.5 quintillion bytes of data is created each day [1]. Due to the huge volume of data created every second, new concepts such as the age of information (AoI) has been established to access the latest version of data [2]. According to the well-known 4V's characteristics of big data, the big data applications need to deal with a very large Volumes of data, which are in Various types, and their Velocity is more than conventional data, while the data Veracity is not confirmed [3].

To process data with the aforementioned characteristics, data should frequently move between storage systems and memory units of the application servers. This high-cost data movement imposes energy consumption and degrades the performance of big data applications. To overcome this issue, data processing has moved toward a new paradigm: "move process to data" rather than moving high volumes of data. Figure 1 compares the traditional "data move to process" concept versus the "move process near data" paradigm.

In the modern clusters, nodes are connected through a low-latency and power-hungry interconnect network such as InfiniBand [4] or Myrinet [5]. In such systems, moving data can be more expensive than processing data [6], and moving the data through an interconnect network is much more costly. In fact, accessing and transferring stored data from storage systems is a huge barrier toward reaching compelling performance and energy efficiency. To deal with this issue, some frameworks such as Hadoop [7] provide mechanisms to process data near where they reside. In other words, these frameworks push process closer to data to avoid massive data movements between storage systems and application servers.

In-storage processing (ISP) is the concept of pushing the process closer to data in its ultimate boundaries. This technology proposes utilizing embedded processing engines inside the storage devices to make them capable of running user applications in-place, so data do not need to leave the device to be processed. This technology has been around for a few years. However, the modern solid-state drives (SSDs) architecture, as well as

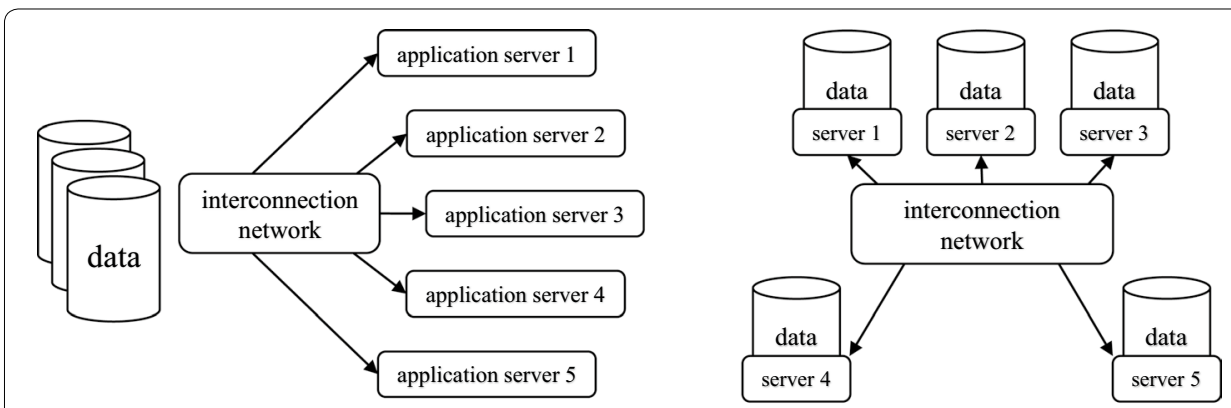

Fig. 1 "Data move to process" and "move process near data" comparison 
the availability of powerful embedded processors, make it more appealing to run user applications in-place. SSDs deliver higher data throughput in comparison to hard disk drives (HDDs). Additionally, in contrast to the HDDs, the SSDs can handle multiple I/O commands at the same time. These differences have motivated researchers to modify applications based on the modern SSD architectures to improve the performance of the applications significantly [8].

The SSDs contain a considerable amount of processing horsepower for managing flash memory array and providing a high-speed interface to host machines. These processing capabilities can provide an environment to run user applications in-place. Based on the reasons mentioned above, this paper focuses on the modern SSD architecture, and in the rest of the paper, computational storage device (CSD) refers to an SSD capable of running user applications in-place.

In an efficient CSD architecture, the embedded ISP engine has access to the data stored in flash memory array through a low-power and high-speed link. Thus, the deployment of such CSDs in clusters can increase the overall performance and efficiency of big data and high-performance computing (HPC) applications. The CSDs and the Hadoop platform both emerged based on the concept of "move process closer to data," and they both can be deployed simultaneously in a cluster. This combination can minimize the data movement and increase efficiency for running big data applications in a Hadoop cluster. However, HPC clusters that are not developed based on the Hadoop platform can still utilize CSDs to improve performance and energy consumption.

Processing user applications inside storage units without sending data to the host processor seems appealing; however, proposing a flexible and efficient CSD architecture has the following challenges.

1. ISP engine: SSDs come with multiple processing cores to run the conventional SSD controller routines. These cores can be utilized for running user applications as well. However, there are two major problems in utilizing the existing SSD cores for instorage processing. First, these cores are usually busy doing normal SSD operations and using them for running user applications can negatively affect the $\mathrm{I} / \mathrm{O}$ performance of the drive. Second, these processing engines are usually real-time cores such as ARM Cortex-R series, which limits the category of the applications that can efficiently run on these cores; also, user applications need major modifications to be able to run on these cores.

2. Host-CSD communication: In a CSD architecture, there should be a mechanism for the communication between host and CSD to submit ISP commands from the host to CSD and receive the results. The conventional SSDs have one physical link connected to the host, which is designed for transferring data. There are many protocols for sending data through this link such as SATA [9], SAS [10], and NVMe over PCIe [11]. None of these protocols are designed for sending ISP commands and results. Thus, this is the responsibility of the CSD designer to provide an ISP communication protocol between host and CSD.

3. Block-level or filesystem-level: An embedded processing engine inside a CSD has access to the raw data stored on the flash, but the filesystem metadata is in control of the host. Therefore, data access inside the storage unit is limited to the block-level 
data, and any application running in-place should not expect to be able to access the filesystem-level data. This limits the type of programming models available for developing ISP-enabled applications, and also the reuse of other applications. Hence, the CSD designer should provide a mechanism to access the filesystem metadata inside the ISP engine so that applications that are running in-place can open files, process data, and finally create output files to write back the results.

4. Host-CSD data synchronization: In a CSD-equipped host, both host and ISP engine have access to the same flash memory array. In such a system, without a synchronization mechanism, these two machines may not be able to see each other's modifications and could result in data corruption.

5. CSD as an augmentable resource: Adding CSDs to a host machine should not limit the host from accessing the data and processing it. The processing horsepower of the CSDs should be an augmentable resource so that the host and CSDs could process data simultaneously. If processing an application in CSD interferes with the host's access to the data, this would dramatically decrease the utilization of the host and the efficiency of the whole system. A well-designed CSD architecture allows the host to access data stored in the flash memory at any time.

6. Adaptability: CSDs should provide a flexible environment for running different types of applications in-place. If the ISP engine of a CSD supports very limited programming languages or needs users to rewrite the application based on a specific programming model, this can affect the adoptability of the CSD significantly.

7. Distributed in-storage processing: A single CSD with limited processing horsepower may not be able to enhance an application's performance significantly, so in many cases, there should be multiple CSDs orchestrating together to deliver compelling performance improvement. For doing such distributed processing, CSD designers need to provide the required tools for implementing a distributed processing environment among multiple CSDs.

8. ISP for high-performance computing: Highly demanding applications such as HPC algorithms can potentially run inside CSDs. However, to serve this class of applications properly, CSDs should be able to boost their performance for some specific applications. In other words, the CSD architecture should be customizable to run some applications in an accelerated mode. Hence, CSD designers are required to provide ASIC- or FPGA-based accelerators to run highly demanding applications satisfactorily.

In this paper, we propose an efficient CSD platform, named Catalina, which addresses all the challenges mentioned above and is flexible enough to run HPC applications in-place or playing the role of an efficient DataNode in a Hadoop cluster. Catalina is equipped with a full-fledged Linux operating system (OS) running on a quad-core ARM 64-bit application processor which is dedicated to run user applications in-place. Catalina utilizes a Xilinx Zynq Ultrascale+ MPSoC [12] as the main processing engine and contains ASIC-based processing engines such as Neon single instruction multiple data (SIMD) engines which can be utilized to accelerate HPC applications that run in-place. Catalina is an augmented resource which can be deployed in Hadoop clusters so that MapReduce applications can simultaneously run on both conventional nodes as well as Catalina 
CSDs [13]. This property makes it easier to adopt this technology in Hadoop clusters. For the proof of concept, we developed a fully functional Catalina CSD prototype and built a platform equipped with 16 Catalina CSDs.

The contributions of this paper can be summarized as follows:

- Proposing the deployment of computational storage devices in clusters, and end-toend integration of CSDs in clusters for running Hadoop MapReduce and HPC applications.

- Describing the hardware and software architecture of Catalina as the first computational storage device which is seamlessly adoptable in Hadoop and MPI-based clusters without any modification in the cluster's software or hardware architecture.

- Prototyping Catalina CSD and developing a platform equipped with 16 Catalina CSDs to practically evaluate the benefits of deployment of computational storage devices in the clusters.

- Exploring the performance and energy consumption of CSD-equipped clusters using Intel HiBench Hadoop benchmark suite as well as 1D-, 2D-, and 3D-DFT operations on large datasets as the HPC benchmarks.

The rest of this paper is organized as follows: "Background" section reviews subjects covered in this paper, such as the modern SSD architecture, ISP technology, and Hadoop platform architecture. In "Method" section, we present the hardware and software architecture of Catalina CSD and describe the unique features that make it capable of seamlessly running a wide spectrum of applications in-place. This section also illustrates the developed Catalina prototype and how it can be deployed in clusters to run Hadoop MapReduce and HPC applications. "Results and discussion" section investigates the benefits of deploying the proposed solution for running Hadoop MapReduce and HPC applications on clusters. This section demonstrates how increasing the number of Catalina CSDs improves energy consumption and performance of the applications. "Related works" section reviews the related works in the field of in-storage processing, and "Conclusion" section concludes the paper with some final remarks.

\section{Background}

The storage system, where data originally reside, plays a crucial role in the performance of applications. In a cluster, the data should be read from the storage system to memory units of the application servers to be processed. As the size of data increases, the role of the storage system becomes more important since the nodes need to talk to the storage units more frequently to fetch data and write back the results. Recently, cluster architects have considered solid-state drives (SSDs) over hard disk drives (HDDs) as the major storage units in modern clusters due to better power efficiency and higher data transition rate [14].

SSDs use NAND flash memory as storage media. NAND memory units are faster and more power-efficient than magnetic disks that are used in HDDs, so SSDs are considered more efficient than HDDs. However, this efficiency comes with complexity in the design and implementation of SSDs, where a multi-core controller is needed to manage the flash memory array. On the other hand, SSDs usually provide a high-speed interface 
to communicate with the host, such as NVMe over PCIe [11]. Implementing such interfaces require embedding more processing horsepower inside SSDs. A modern SSD controller is composed of two main parts: 1 -front-end (FE) processing engine providing high-speed host interface protocol such as NVMe/PCIe, and 2-back-end (BE) processing engine which deals with flash management routines. These two engines talk to each other to accomplish host's I/O commands.

A NAND flash memory chip is a package containing multiple dies. A die is the smallest unit of flash memory that can independently execute $\mathrm{I} / \mathrm{O}$ commands and report status. Each die is composed of a few planes, and each plane contains multiple blocks. Erasing is performed at the block-level, so a flash block is the smallest unit that can be erased. Inside each block, there are several pages which are the smallest units that can be programmed and written. The key point in this hierarchical architecture is the programmable unit versus the erase unit. The NAND flash memory can be programmed in page-level, which is usually 4 to $16 \mathrm{kB}$, while the erase operation cannot be done on a smaller segment than a block which is few megabytes of memory. Also, each flash block can be erased for a finite number of times, and flash blocks wear as erase operations take place, so it is important to balance the number of erase operations among all the flash blocks of an SSD. The process of leveling the number of erase operations is called wear leveling. In addition, the logical address exposed to the host is different from physical block addresses, so there are multiple tables for logical and physical address translation. The flash translation layer (FTL) is composed of all the routines needed to manage flash memory arrays such as logical block mapping, wear leveling, and garbage collection. Overall, the BE processing subsystem of a modern SSD architecture handles FTL, while the FE subsystem provides protocols to communicate with the host. The details of FTL processes and NVMe protocol are out of the scope of this paper. The high-level architecture of a modern SSD is demonstrated in Fig. 2.

The Webscale data center designers have been trying to develop storage architectures that favor high-capacity hosts, and this fact is highlighted at OpenCompute (OCP) by Microsoft Azure and Facebook that call for up to 64 SSDs attached to each host [15].

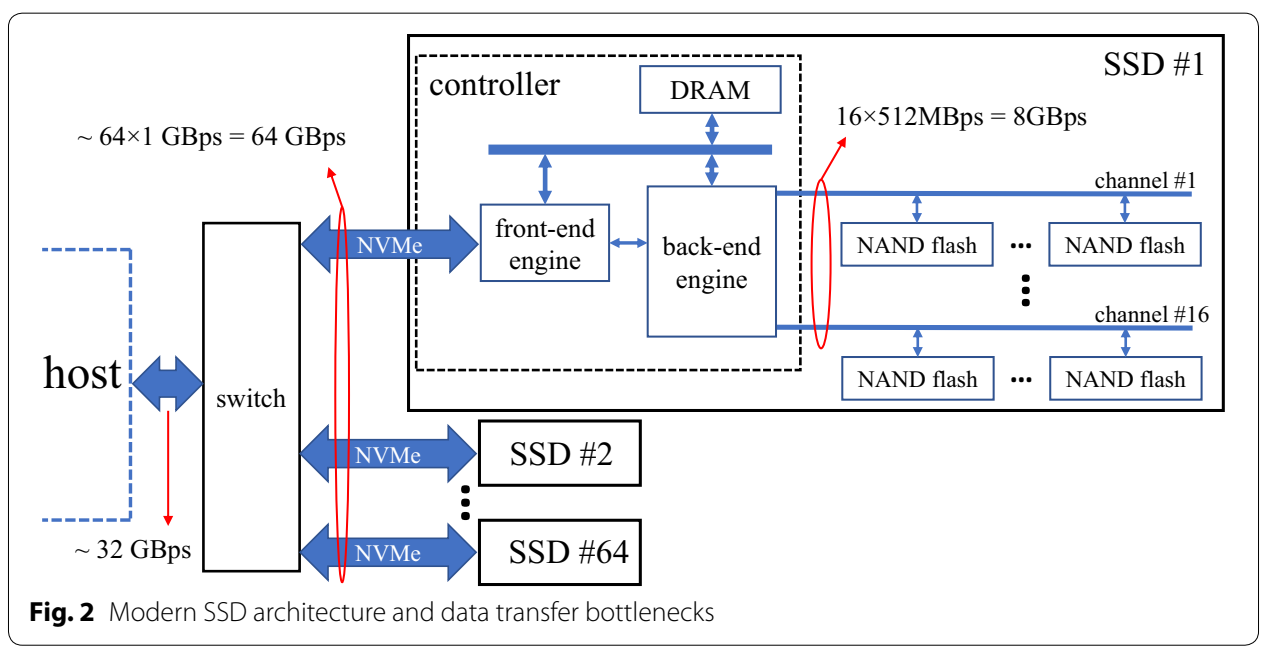


In Fig. 2, such a storage system is shown where 64 SSDs are attached to a host. For the sake of simplicity, only the details of one SSD are demonstrated. Modern SSDs usually contain 16 or more flash memory channels which can be utilized concurrently for flash array I/O operations. Considering $512 \mathrm{MBps}$ bandwidth per channel, the internal bandwidth of an SSD with 16 flash memory channels is $8 \mathrm{GBps}$. This huge bandwidth decreases to about $1 \mathrm{GBps}$ due to the complexity of the host interface software and hardware architecture. In other words, the accumulated bandwidth of all internal channels of the 64 SSDs reaches the multiplication of the number of SSDs, number of channels per SSD, and $512 \mathrm{MBps}$ (bandwidth of each channel) which is equal to $512 \mathrm{GBps}$. While the accumulated bandwidth of the SSDs' external interfaces is equal to 64 multiply by 1 GBps (the host interface bandwidth of each SSD) which is 64 GBps. However, In order to talk to the host, all SSDs required to be connected to a PCIe switch. Hence, the available bandwidth of the host is limited to $32 \mathrm{GBps}$.

Overall, there is a $16 \times$ gap between the accumulated internal bandwidth of all SSDs and the bandwidth available to the host. In other words, for reading $32 \mathrm{~TB}$ of data, the host needs 16 min while internal components of the SSDs can read the same amount of data in about $1 \mathrm{~min}$. Additionally, in such storage systems, data need to continuously move through the complex hardware and software stack between hosts and storage units, which imposes a considerable amount of energy consumption and dramatically decreases the energy efficiency of large data centers. Hence, storage architects need to develop techniques to decrease data movement, and ISP technology has been introduced to overcome the aforementioned challenges by moving process to data.

In a traditional CPU-centric scheme, data always move from storage devices to CPU to be processed, and this mechanism, which is inherently limited by the von Neumann bottleneck, is the root cause of the challenges above, especially when many SSDs are connected to a host. ISP technology proposes a contrary approach to push the "move process to data" to its ultimate boundaries where a processing engine inside storage unit takes advantage of high-bandwidth and low-power internal data links and processes data inplace. In fact, "move process to data" is the concept that leads to the emergence of both ISP and distributed processing platforms such as Hadoop. Later in this section, it will be described how the Hadoop platform and ISP technology can simultaneously work together in a cluster.

The ISP technology minimizes the data movements in a cluster and also increases the processing horsepower of the cluster by augmenting power-efficient processing engines to the whole system. This technology can potentially be applied to both HDDs and SSDs; however, modern SSD architecture provides better tools for developing such technologies. The SSDs which can run user application in-place are called computational storage devices (CSDs). These storage units are augmentable processing resources, which means they are not designed to replace the high-end processors of modern servers. Instead, they can collaborate with the host's CPU and augment their efficient processing horsepower to the system.

It is noteworthy that CSDs are fundamentally different than object-based storage systems such as Seagate Kinetic HDDs [16], which transfer data at the object-level instead of block-level. The object-based storage units can receive objects (i.e., image files) from a host, store them, and at a later time, retrieve the object back to the host using an object 
ID. Consequently, the host does not require to maintain metadata of block addresses of the object. On the other hand, CSDs can run user applications in-place without sending data to a host. There is a vast literature in this field that proposed different CSD architectures and investigated the benefits and challenges of deploying CSDs for running applications in-place. In "Related works" section, we will review the important works in this field.

A while ago, when the cost of data movement was insignificant in comparison with the computational cost, there could be a centralized storage system, and other hosts could send requests to it to fetch data blocks. With this mechanism and today's volume of data, a data-intensive application requires large amounts of data to be fetched from the storage system, and such huge data movements drastically increase energy consumption. With the emergence of big data, the storage system can no longer be centralized, and the traditional approaches come short of satisfying super-scale applications' demands, which call for scalable processing platforms. To answer these demands, distributed processing platforms such as Hadoop are proposed to process data near where they reside [17].

Hadoop has emerged as the leading computing platform for big data analytics and is the backbone of hyperscale data centers [18], where hundreds to thousands of commodity servers are connected to provide service to clients. The Hadoop distributed processing platform consists of two main parts, namely Hadoop filesystem (HDFS) and MapReduce engine. HDFS is the underlying filesystem of the Hadoop platform, and it is responsible for partitioning the data to blocks and distribute the data blocks among nodes. HDFS also generates a certain number of replicas of each block to make the system resilient against storage or node failures. It consists of a NameNode host which takes care of filesystem metadata such as the location of the data block and status of the other nodes, and multiple DataNodes hosts that store the data blocks.

On top of HDFS, MapReduce platform takes advantage of the partitioned data and runs map and reduce functions and orchestrates the cluster nodes to run distributed applications while data movements are minimalized. The Apache MapReduce 2 (YARN) is one of the well-known MapReduce platforms [19]. YARN agents manage the procedure of running map tasks, performing shuffle and sort, and running the reduce functions to generate the output of the MapReduce application. The most important agents in the YARN framework are global resource manager $(R M)$, one node manager $(N M)$ per processing node, and an application master $(A M)$ per MapReduce application.

The $R M$ has a list of all the resources available in the cluster and manages the highlevel resource allocations to MapReduce applications. On the other hand, NMs that run on the processing nodes manage the local hosts' resources. The $R M$ regularly talks to the $N M$ s to manage all the resources and poll the status of the nodes. For each MapReduce application, there is an $A M$ to observe and manage the progress of the application. Regularly, $R M$ runs in the same node that HDFS NameNode runs (head node), and NMs run together with the HDFS DataNodes. Figure 3 shows a high-level overview of a MapReduce application on a Hadoop platform.

In the Hadoop environment, data should initially be imported to the HDFS. This process includes partitioning the input data to data blocks and storing them in the DataNodes. At this point, the data blocks are ready to be processed in a distributed 


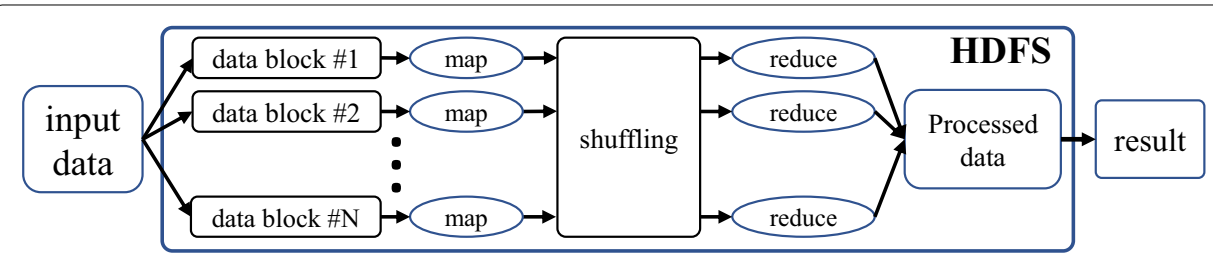

Fig. 3 MapReduce process on Hadoop cluster

fashion. Since map functions preferably process the local data blocks, the MapReduce framework is known for moving process closer to data in order to improve the power efficiency and performance of the applications.

A MapReduce application targets a set of input data blocks as well as the user-defined map and reduce functions. The procedure starts by running the map function on the targeted data blocks. Map function instances run concurrently on the DataNodes, consume data blocks, and produce a set of key/value pairs to be used as the input of the reduce function. These intermediate key/value pairs are stored locally on the DataNodes that execute the map function and should be shuffled, sorted, and transferred to the nodes which run the reduce tasks. The Hadoop framework stores the output of the reduce function in HDFS, and subsequently, it can be imported to a host's local filesystem.

The Hadoop MapReduce platform provides an efficient environment for processing large datasets in a distributed fashion. However, there are research papers in the literature that optimize Hadoop MapReduce platform for different workloads. Generally, HDFS partitions data to fixed-size 64 or 128 megabytes data blocks. The data-intensive MapReduce applications send numerous I/O requests to the Hadoop I/O scheduler, and this leads to a long queue of $\mathrm{I} / \mathrm{O}$ requests in the scheduler, and consequently, a substantial increase in the data latency. Bulk I/O dispatch (BID) is proposed as a Hadoop I/O scheduler that is optimized for data-intensive MapReduce applications and can improve the access time of such applications significantly [20].

In some big data applications, the data that are generated in a processing node will be consumed by the same node in the near future. The lineage-aware data management (LDM) is proposed for Hadoop-based platforms to exploit this data locality to decrease the network footprint [21]. LDM develops a data block graph which is used to characterize the future data usage pattern. Using this information, it defines tier-aware storage policies for the Hadoop nodes to mitigate the impact of data dependency.

The Hadoop strategy of "processing data close to where they reside" is completely aligned with the ISP paradigm [22]. So, they can fortify each other's benefits when both are deployed concurrently in a cluster. In other words, Hadoop-enabled CSDs can play both roles of fast storage units for conventional Hadoop DataNodes and ISPenabled DataNodes simultaneously, resulting in augmentation of processing horsepower of the CSDs to the Hadoop cluster.

Although CSDs can improve the overall performance of a MapReduce application by augmenting their processing engine to the Hadoop framework, this is not the primary advantage of deploying CSDs in the clusters. Potentially, increasing the total horsepower of a cluster can be achieved by adding more commodity nodes to a 
cluster. What makes CSDs distinguishable is, in fact, utilizing the high-performance and power-efficient internal data links of the modern SSD architecture for running Hadoop MapReduce applications.

On the other hand, well-designed CSDs can be deployed to run HPC applications inplace. However, CSDs need to deliver a compelling performance when running HPC applications; otherwise, it is hard to justify the complexity of deploying CSDs in the clusters while their performance improvement is not satisfactory. In this paper, we argue that CSDs can considerably improve the performance of HPC applications when they utilize ASIC-based accelerators such as Neon advanced SIMD engines.

\section{Methods: Catalina CSD for MapReduce and HPC applications}

We mentioned the challenges for proposing a well-designed CSD architecture in "Introduction" section. Based on these challenges, we set eight design goals to propose an efficient and flexible CSD architecture. The design goals are as follows: 1-A desired CSD architecture should avoid using real-time processors that are originally intended to run conventional flash management routines for running user applications in-place. Instead, it should contain an ISP-dedicated application processor. 2-There should be a TCP/IP link between the host and the ISP engine, allowing the applications running on the host and CSD to communicate with each other. 3-The ISP engine should have access to the filesystem metadata. 4-Since both host and ISP engine have access to the flash memory concurrently, there should be a synchronization mechanism. 5-Both host and ISP engine should be able to interact with the flash storage simultaneously. 6 -There should be an OS running inside the CSD. This OS provides a flexible environment to run a vast spectrum of applications in-place. 7-The desired CSD should support the distributed processing platforms such as Hadoop and message passing interface (MPI). 8-The CSD should have potentials to implement ASIC- and FPGA-based accelerator engines to run CPU-intensive applications in-place with a compelling performance.

In this section, we describe the hardware and software architecture of Catalina, which is designed to satisfy all the design goals mentioned above. This section is composed of four subsections. In the first subsection, different hardware components of Catalina are described, and we discuss how they work together. The second subsection defines Catalina software layers that make it possible to send ISP commands, processing data in-place, and writing the results back. The third subsection demonstrates the fully functional Catalina prototype used to investigate the benefits of deploying CSDs in the clusters. The last subsection demonstrates how multiple Catalina CSDs can be deployed to run Hadoop MapReduce and HPC applications.

\section{Hardware architecture}

Catalina CSD is developed based on Xilinx Zynq Ultrascale+ MPSoC [12]. This device is composed of two subsystems, namely programmable logic (PL) and processing system (PS). The PS is an ASIC-based processing subsystem, including a quad-core ARM Cortex-A53 64-bit processor equipped with Neon SIMD engines and floating-point units, two ARM Cortex-R5 real-time processors, DRAM controller, as well as other interconnect and data movement components. Adjacent to PS, there is the PL subsystem which is an FPGA that can be utilized for implementing different components of the CSD 
controller such as the host and flash memory array interfaces. These two subsystems are packaged in one chip with multiple data links connecting them for high-performance and power-efficient intra-chip data transfers. These two subsystems together provide a proper platform for implementing conventional SSD routines as well as running user applications in-place.

Figure 4 shows the Catalina architecture, implemented using Xilinx Zynq Ultrascale+ MPSoC. On the PL subsystem, there are three conventional components of the controller which are host NVMe/PCIe interface, error correction unit, and the flash memory interface. The host interface is responsible for sending and receiving the $\mathrm{NVMe} / \mathrm{PCI}$ packets from the host and check the integrity of the payloads. Soft errors can potentially happen in different parts of a device, and SSDs are no exceptions. These errors can happen in the SSD controller and the flash memory packages. There are research works that investigate the effect of the soft errors on the SSD controller and the availability and reliability of the storage systems [23]. On the other hand, for addressing the soft errors on the flash memory packages, the error correction unit is utilized to correct the data errors.

The flash memory interface controls the flash memory channels. In Fig. 4, each flash channel is connected to a set of flash memory packages. Design and implementation of these three components require a considerable amount of engineering resources; however, they are common among all conventional SSDs. We skip the detailed architectures of these components since they are out of the scope of this paper.

On the PS subsystem, there are two ARM Cortex-R5 real-time processors that are used for controlling the components implemented in the PL subsystem as well as running the flash translation layer (FTL) routines. In fact, the conventional firmware routines run on these two real-time processors. One of the real-time processors runs the FE firmware, which controls the host interface module and interprets the host's I/O commands. The other ARM Cortex-R5 processor runs the BE firmware, which is responsible for controlling the error correction and flash interface units. The BE firmware also runs other essential FTL routines such as garbage collection and wear-leveling. The FE and

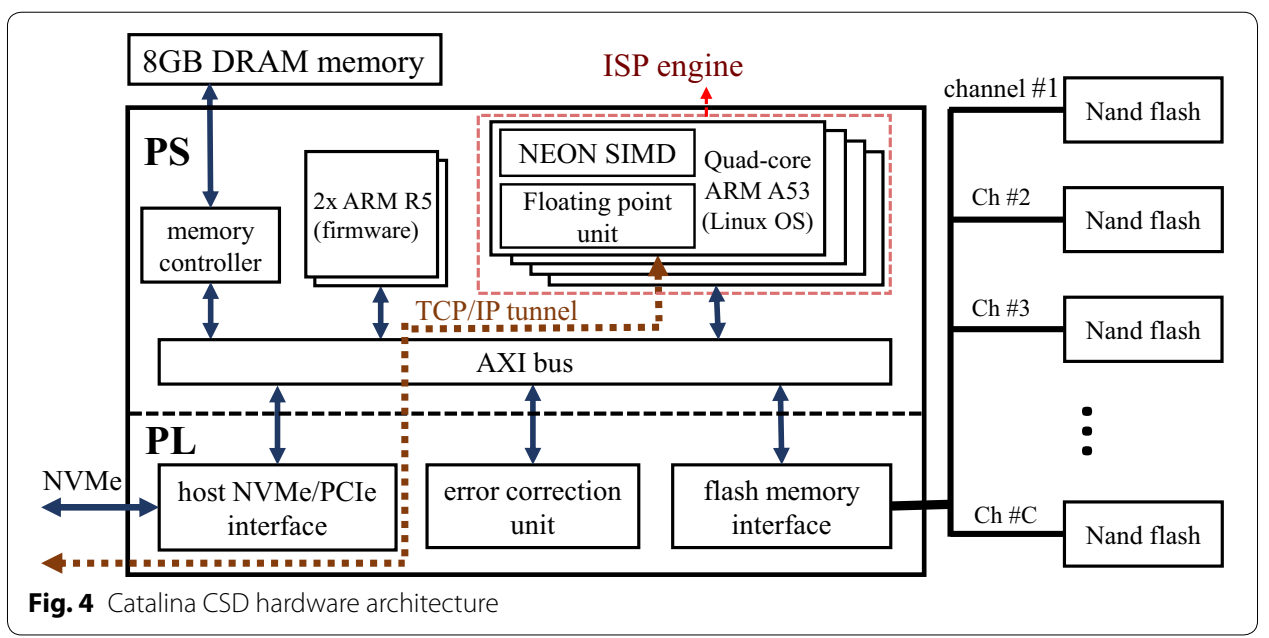


BE firmwares work together to receive the host's I/O commands, interpret them, and execute flash read/write operations.

All of the components mentioned above are common among conventional SSDs; however, Catalina is equipped with a unique ISP subsystem. This subsystem is dedicated to running user applications in-place. It contains a quad-core ARM Cortex-A53 processor which is equipped with Neon SIMD engines and floating-point units (FPUs). The quadcore processor is capable of running a vast spectrum of applications, while Neon SIMD engines can increase the performance of HPC applications. Overall, the quad-core Cortex-A53 processor is the main ISP engine of Catalina, and Neon SIMD engines and the FPUs can accelerate user applications that run in-place.

As Fig. 4 demonstrates, both the ISP engine and the two Cortex-R5 real-time processors which run the conventional flash management routines are connected via an internal Xilinx advanced extensible interface (AXI) bus. The shared AXI bus makes it possible to transfer data between the BE firmware and the ISP engine efficiently. In other words, the ISP engine can bypass the whole NVMe hardware and software stack and access the data stored in the flash memory array directly by communicating to the BE firmware. There is also an 8 GB DRAM memory connected to the AXI bus, which is shared among all the processing units.

\section{Software stack}

The Catalina software stack is designed to achieve the goals we set earlier in this section. The most important part of the software components is an OS running inside the ISP engine. Therefore, we have ported a full-fledged Linux OS running on the quad-core ARM Cortex-A53 processor. Each core of the quad-core processor has a level-one $32 \mathrm{kB}$ instruction cache and a $32 \mathrm{kB}$ data cache. The ARM processor also contains a $1 \mathrm{MB}$ level-two cache, which is used by all the cores. These caches together with the memory unit and the non-volatile flash memory compose a memory hierarchy. The Catalina OS governs the memory hierarchy for data coherency and integrity. Overall, the OS is a flexible environment for running user applications in-place as well as implementing other layers of the software stack.

Running an OS inside the CSD comes with complexity and processing overhead. The first challenge to port an OS inside the CSD is to boot the OS together with the conventional flash management firmware. At the boot time, the OS and the firmware should boot up concurrently and complete a handshake phase. This procedure adds a considerable amount of complexity to the design and manufacturing of the CSD. We have successfully implemented this dual-boot procedure, and the users do not have any concerns regarding this challenge.

On the other hand, the ISP subsystem consumes a portion of the processing budget for running the OS routines. Since the processing budget of the ISP engine is limited, the OS should not consume a big portion of the resources. We have measured the OS routines overhead in different states of the ISP subsystem, and our results showed that the processing overhead of running the OS is at most $2 \%$ of the total processing budget of the Catalina CSD. Figure 5 demonstrates the architecture of the software layers and how they make it possible to run distributed applications in-place. 
Our first design, named Compstor [24], had a custom-developed software stack, and it was unable to be utilized in the distributed processing environments. This problem is addressed in Catalina design by adopting standard software stacks such as MPI and Hadoop MapReduce [25]. In Fig. 5 there is a cluster of $M$ hosts connected to a TCP/IP interconnect, and the host 1 is attached to $N$ Catalina CSDs via a PCIe switch. In this figure, the lowest layer of the software stack is the BE firmware, which implements the FTL procedures. The BE firmware serves both FE firmware which talks to the host via NVMe protocol and a block device driver implemented in the kernel space of the ISP engine's OS. The block device driver issues flash $\mathrm{I} / \mathrm{O}$ commands directly to the $\mathrm{BE}$ firmware, so the data link through the block device driver bypasses the NVMe/PCIe software and hardware stack. The block device driver also makes it possible to mount the flash storage inside the Catalina OS. It allows a user application running in-place to have filesystem-level access to the data stored in the flash memory array via a highperformance and low-power internal data link.

On the other hand, the ISP engine should also provide a link between applications that run in-place and applications running on the host, so in addition to the block device driver, we have implemented a TCP/IP tunnel through NVMe protocol to transfer TCP/IP packets between the applications running on the host and the applications running inside Catalina. We have utilized NVMe vendor-specific commands to packetize TCP/IP payloads inside the NVMe packets (TCP/IP tunnels through

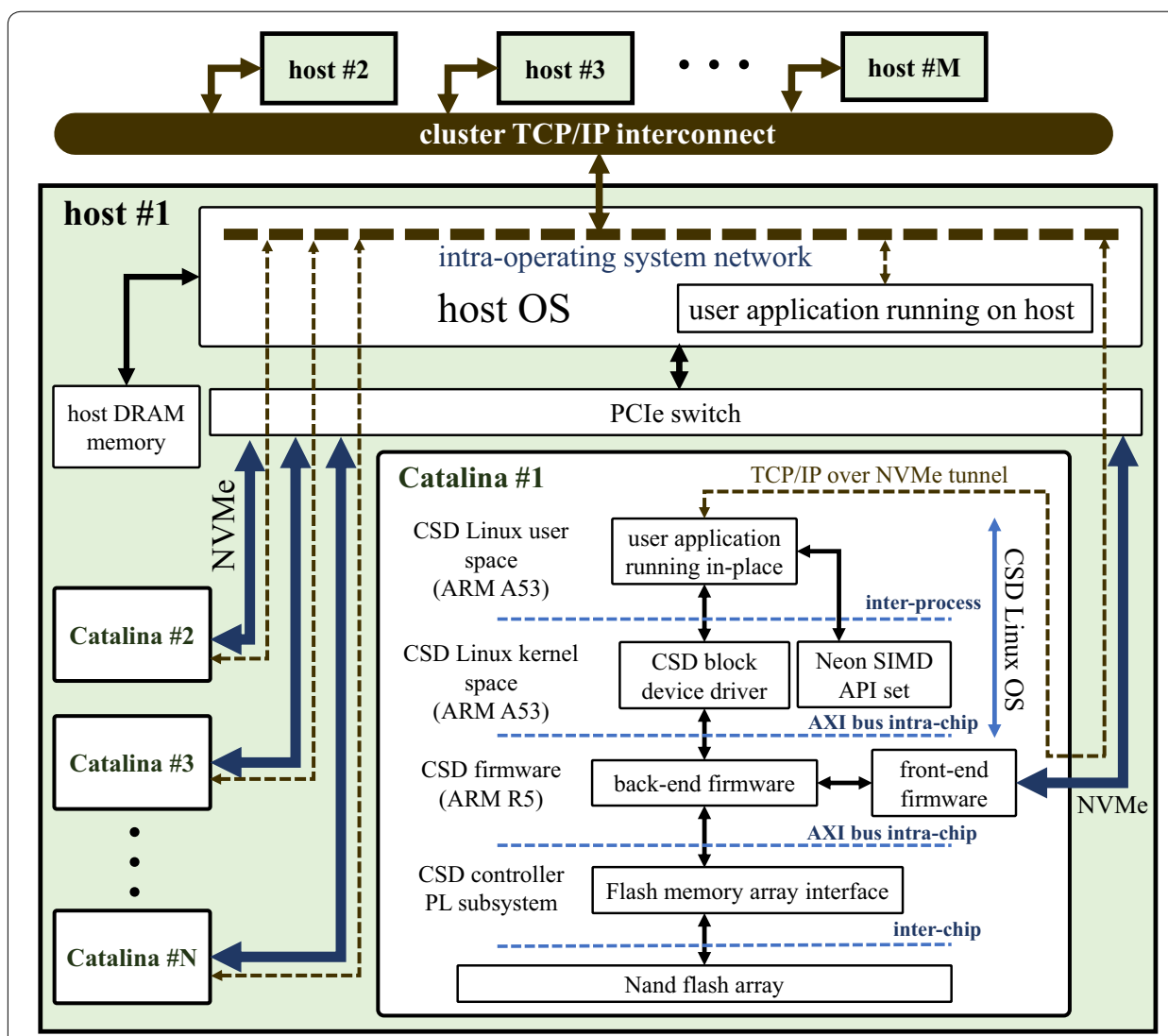

Fig. 5 Catalina CSD software stack 
NVMe are demonstrated in Fig. 5 by dashed lines). A software layer implemented on both host OS and the Catalina OS provides the tunneling functionality. Since distributed platforms such as Hadoop MapReduce [17] and MPI [26] are based on TCP/ IP connection, this link plays a crucial role in running distributed applications. As shown in Fig. 5 , all the $N$ Catalina CSDs that are attached to the host 1 can concurrently communicate with applications running on the host.

It is worth mentioning that by using Linux TCP/IP packet routing tools, we can create an internal network in the host OS, and reroute the packets sent or received by the Catalina CSDs to the other hosts attached to the TCP/IP interconnect (see Fig. 5). Alternatively stated, considering several hosts that are connected via a TCP/IP interconnect, and each of them is equipped with multiple Catalina CSDs, the hosts, as well as all the CSDs attached to them, can communicate to each other via a TCP/IP network. Such CSD-equipped cluster architecture benefits from the efficient ISP capabilities of Catalina CSDs to run distributed applications. In fact, the proposed CSD architecture is an augmentable processing resource that is adoptable in the cluster without any modifications in the underlying Hadoop or HPC platforms.

Also, the user applications that run in-place have access to Neon SIMD engines via a set of application programming interfaces (APIs) provided by the Catalina OS. Using these APIs, user applications can potentially be accelerated by the Neon SIMD engine. Overall, the user applications have access to three unique tools, including 1-a highspeed and low-power internal link to the data stored in the flash memory, 2-a TCP/ IP link to the applications running on the host, 3-a set of APIs to utilize Neon SIMD engines.

The last layer of the software stack is the synchronization layer between the host and Catalina OSs. These two OSs can access the data stored in the flash memory array at the filesystem-level and concurrently mount the same storage media, which is a problematic behavior without a synchronization mechanism. Since Catalina contains a full-fledged Linux OS and there is a TCP/IP connection to the host, to address the synchronization issue, we have utilized the Oracle cluster filesystem 2nd version (OCFS2) [27] between the host and the CSD. Using OCFS2, both the host and Catalina CSD can issue flash I/O commands and mount the shared flash memory natively. This is the main difference between OCFS2 and network filesystem (NFS). In NFS, only one node mounts the shared storage natively, and other nodes use a network connection to access the shared storage, so NFS limits the data throughput and also suffers from the single point of failure problem. On the other hand, using OCFS2, all nodes can mount the storage natively.

\section{Catalina prototype}

To prove the feasibility of the proposed computational storage solution and also investigate the benefits of deploying Catalina CSDs in clusters, we have designed and manufactured a fully functional prototype of Catalina which completely aligns with the hardware and software architecture that has been described in the previous subsections. Figure 6 shows the Catalina CSD prototype. The CSD controller implemented on a Xilinx Zynq Ultrascale+ MPSoC, as well as the NAND flash packages, are shown in this figure. 


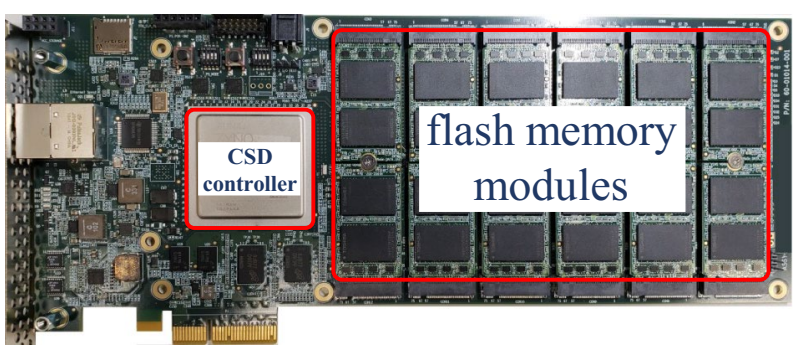

Fig. 6 Catalina CSD prototype

Table 1 Catalina prototype hardware specifications

\begin{tabular}{llc}
\hline FTL and host interface engine & Processing units & $\begin{array}{c}\text { Two ARM R5 proces- } \\
\text { sors @600 MHz }\end{array}$ \\
& & FPGA @250 MHz \\
& Nost interface & NVMe over PCle Gen3 \\
In-storage processing engine & Processing units & Quad-core ARM \\
& & Cortex-A53 with \\
& & Neon SIMD engines \\
& TCP/IP ove NVMe \\
Shared & Host interface & $8 \mathrm{~GB}$ \\
\hline
\end{tabular}

The CSD controller is composed of the PS and PL subsystems that implement the two processing engines of the Catalina CSD, namely the conventional FTL and host interface engine and the ISP engine. The hardware specifications of the Catalina prototype separated for these two engines come in Table 1.

The prototype of Catalina is able to execute the host's I/O commands and also provides a straightforward mechanism for offloading user applications to the CSD via a TCP/IP tunnel through NVMe/PCIe. Considering multiple Catalina CSD prototypes attached to a host, an administrative application on the host can initiate user applications on CSDs while the host and the CSDs' OSs are being synchronized by the OCFS2 filesystem. The user applications can be developed in any language supported by Linux OS, and they can interact with the flash memory at the filesystem-level, similar to when they run on a conventional host machine.

Despite all of the projected benefits of deploying the CSDs, they should be cost-effective to be adoptable in the clusters. After prototyping Catalina, a sensible cost analysis of manufacturing CSDs can be presented. Compared with a regular SSD based on a conventional controller, a CSD should be equipped with more processors to run applications in-place efficiently. Interestingly, according to our observations and also the SSD bill of material analysis $[28,29]$, the major difference between an SSD and a CSD manufacturing costs would be insignificant, since the SSD manufacturing cost is largely dominated by the NAND flash memory. The cost of flash memory chips is about $75 \%$ of the SSD price. ${ }^{1}$ With other miscellaneous costs (such as DRAM, miscellaneous components, and manufacturing costs) that would account for $20-25 \%$ of the SSD price, the controller would account for at most $5 \%$ of the SSD price.

\footnotetext{
${ }^{1}$ data acquired from "https://www.dramexchange.com".
} 


\section{Deploying Catalina in clusters}

Catalina is developed concerning a straightforward deployment in clusters. Since it has all the required features to play the role of a regular processing node, the cluster architects do not have to make major modifications in the underlying platforms for deployment of the Catalina CSDs. Figures 7, 8 illustrate CSD-equipped Hadoop and MPI-based clusters, respectively, where a head node is connected to $M$ host machines, and each of the hosts is equipped with $N$ Catalina CSDs. In such a cluster, all CSDs and conventional nodes are orchestrating together to improve the performance and efficiency of distributed applications.

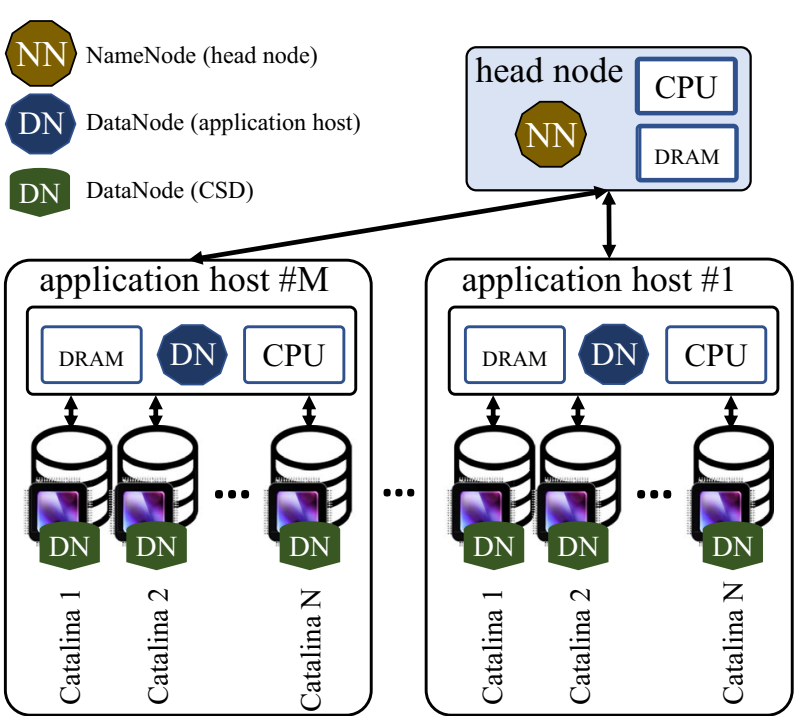

Fig. 7 Catalina CSD-equipped Hadoop cluster

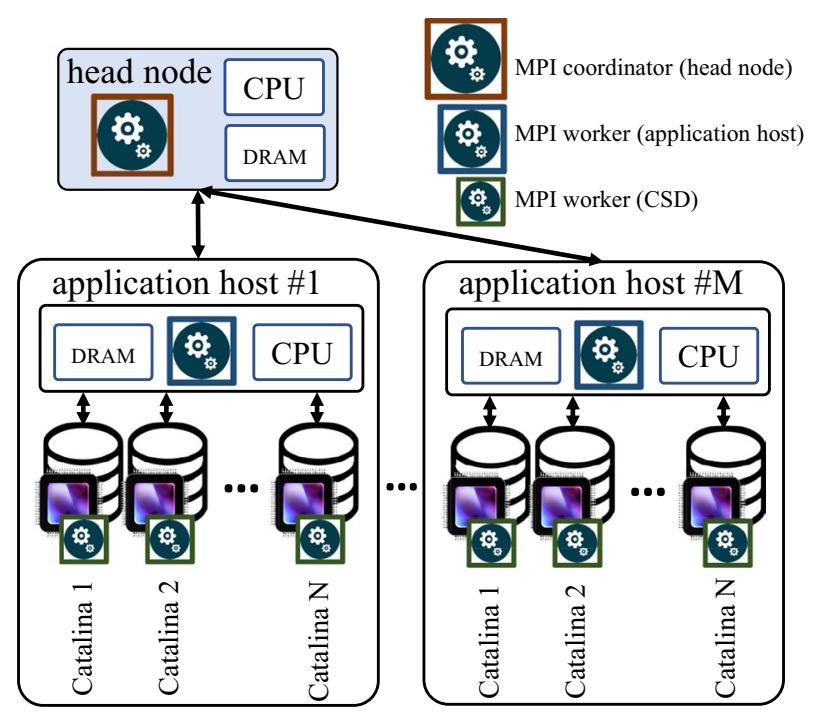

Fig. 8 Catalina CSD-equipped MPI-based cluster 
In the CSD-equipped Hadoop cluster, the head node runs Hadoop NameNode and YARN resource manager $(R M)$, while the hosts and Catalina CSDs run DataNodes and node managers (NMs). In fact, Catalina CSDs play both roles of storage units as well as efficient DataNodes. Since Hadoop implements its filesystem synchronization mechanism, we exceptionally do not need the OCFS2 filesystem for running Hadoop. However, OCFS2 plays an important role in running MPI-based applications.

Figure 8 illustrates a CSD-equipped cluster based on MPI for running HPC applications. In this figure, the head node runs an MPI coordinator while the conventional hosts, and the Catalina CSDs, run the MPI workers. In this MPI-based cluster, each host is attached to N CSDs, and the data stored on the CSDs are shared between the host and CSDs, so the MPI workers on the host and the CSDs have access to the shared data. Thanks to the OCFS2 filesystem, the shared data is simultaneously visible to the host and CSDs at the filesystem-level so that the user can freely distribute the processing load among the hosts and the CSD.

\section{Results and discussion}

As mentioned in the previous section, deploying Catalina CSDs in clusters is straightforward. After attaching the Catalina CSDs to the host and setting the network configurations, the CSDs are exposed to the other hosts in the cluster by their network address (e.g., IP address). From the system-level point of view, the Catalina CSDs are similar to regular processing nodes, and the underlying ISP hardware and software details are invisible to other nodes in the cluster. In this section, we first demonstrate the developed platforms equipped with up to 16 Catalina CSDs and describe how we implemented a CSD-enabled Hadoop and MPI-based clusters on the developed platforms. The second subsection shows the results of running different Hadoop MapReduce and HPC benchmarks and discusses the benefits of deploying Catalina CSDs in clusters.

\section{Experimental setup}

The Catalina CSDs are not designed to compete with the modern hosts based on highend $\times 86$ processors with tens to hundreds of gigabytes of DRAM. Instead, it is presented as a resource that augments the processing horsepower of a system and improves the performance and power efficiency of the applications [25]. However, for getting considerable improvements, we propose attaching multiple Catalina CSDs to host machines. Figure 9 shows the architecture of the developed platform, which contains 16 Catalina CSD prototypes. We built this platform to investigate the benefits of deploying Catalina CSDs in clusters.

This platform is composed of a conventional host (called the head node) and an application host, which is equipped with the Catalina CSDs. These two hosts, along with the Catalina CSDs, form a distributed environment for running Hadoop MapReduce and MPI-based HPC applications. We use the head node exclusively for running Hadoop NameNode and the MPI coordinator to eliminate the load of the administrative tasks on the processing nodes. In other words, the application host and the CSDs are the processing nodes, while the head node is dedicated only for running the administrative tasks.

To extensively investigate the benefits of Catalina CSDs in different environments, we have considered three different configurations for the application host, namely low, 


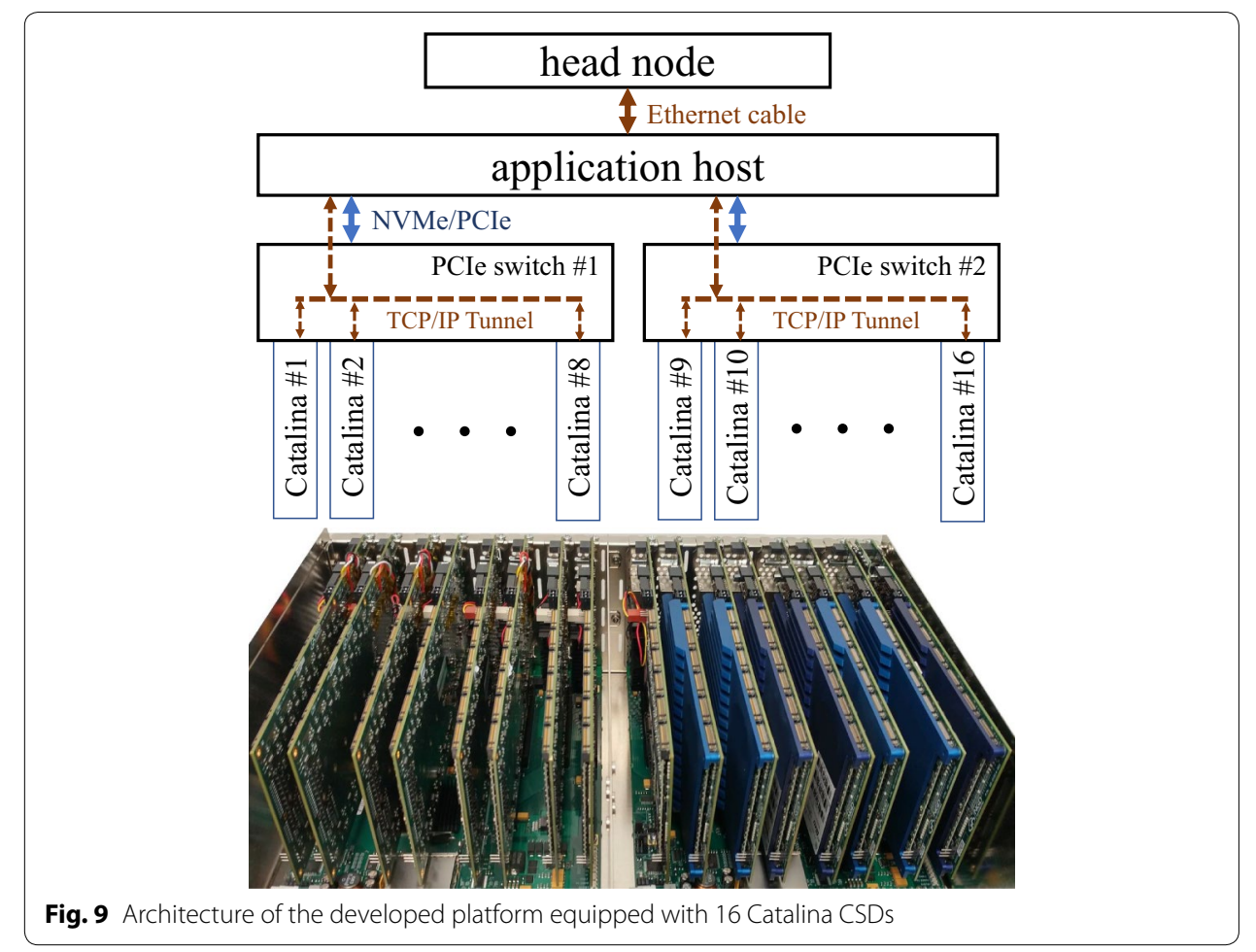

Table 2 Specifications of the hosts in the developed system for running experiments

\begin{tabular}{|c|c|c|c|c|}
\hline \multirow[t]{2}{*}{ Feature } & \multirow[t]{2}{*}{ Head node } & \multicolumn{3}{|c|}{ Application host configurations } \\
\hline & & Low & Medium & High \\
\hline Processor & Xeon E5-2620 v4 & Core i3-8100T & Core i7-7700 & Xeon E5-2620 v4 \\
\hline Memory & 32 GB (DDR 4) & 32 GB (DDR 4) & 32 GB (DDR 4) & 32 GB (DDR 4) \\
\hline Storage & $\begin{array}{l}\text { 4× Samsung } 850 \\
\text { PRO } 1 \text { TB SSD }\end{array}$ & $6 \times$ Catalina CSD & $6 \times$ Catalina CSD & $16 \times$ Catalina CSD \\
\hline In-storage processing & None & $6 \times$ Catalina CSD & $6 \times$ Catalina CSD & $16 \times$ Catalina CSD \\
\hline
\end{tabular}

medium, and high. The specifications for the head node and the different application host's configurations are summarized in Table 2. In order to attach up to 16 Catalina CSDs to the application host, we used a Cubix Xpander Rackmount unit [30] which provides 16 PCIe Gen3 slots. This unit and the attached Catalina CSDs are shown in Fig. 9.

The implementations of the Hadoop and the MPI-based clusters are aligned with the architectures that are shown in Figs. 7, 8. For implementing the Apache Hadoop cluster, we ran Hadoop NameNode and the Yarn resource manager (RM) on the head node, while the DataNodes and the node managers (NMs) run on the application host, and the Catalina CSDs that are attached to the application host. The communication between the head node and the application host is through an Ethernet cable, while Catalina CSDs communicate via the developed TCP/IP through NVMe link.

On the other hand, for running the HPC application based on MPI, we use the head node for running the MPI coordinator application which initiates and organizes the MPI workers that run on the application host and the Catalina CSDs. In this case, the OCFS2 
filesystem synchronizes the filesystems of the Catalina CSDs and the application host, so at any given time the application host can access the whole data stored on all the CSDs directly, while each CSD only has access to its local data.

As previously discussed, all the packets from/to the CSDs are routed inside the application host. This mechanism provides a flexible networking environment without using an extra network switch. However, there is a cost for having such a flexible network. The application host needs to consume a portion of its processing horsepower to route the packets. In our design, for each CSD added to application host, an insignificant amount of the host's CPU is consumed for the routing mechanism. We have measured this networking overhead for different workloads. In highly congested scenarios, the application host consumed at most 3.5\% of its processing horsepower for the routing the TCP/IP packets among CSDs. This overhead is considered in the results in this section.

\section{Benchmarks and results}

This subsection is composed of two parts. First, we describe the targeted Hadoop MapReduce benchmarks and report the performance and energy consumption of running the benchmarks for different configurations to investigate the benefits of running Hadoop MapReduce applications in-place. Then, we show the results for running 1D, 2D, and 3D discrete Fourier transform (DFT) algorithms utilizing the Neon SIMD engines of Catalina CSDs. For reporting the performance, we measure the total elapsed time of running a benchmark on the developed platform for a given configuration. On the other hand, to measure the energy consumption, we use a power meter to measure the power consumption of the platform. Using the logging tool provided by the power meter, we calculate the total energy consumption for running a benchmark. However, we deducted the idle energy consumption from the total calculated energy consumption for all the experiments to eliminate the energy consumption imposed by miscellaneous devices such as the cooling system.

\section{Hadoop MapReduce benchmarks and results}

For running Apache Hadoop MapReduce applications on the developed platform, we have used a subset of the Intel HiBench benchmark suite [31] which includes Sort, Terasort, and Wordcount benchmarks. We believe that extensive experiments on these three benchmarks can show the potentials of CSD architectures running Hadoop MapReduce applications. These benchmarks have been executed on 16 different platform configurations, which are listed in Table 3. In all the experiments, the head node specification is fixed and matches with Table 2, and the number of mappers and reducers tasks are 2000 and 200, respectively.

The application host uses all the attached Catalina CSDs as the storage units (6 CSDs in the low and medium configurations; and 16 CSDs in the high configuration), while in each configuration, a certain number of the ISP engines of the CSDs are enabled to run MapReduce application in-place. This way, the scalability of deploying Catalina CSDs in clusters can be investigated. The data size for Sort, Terasort, and Wordcount benchmarks are $8 \mathrm{~GB}, 1.3 \mathrm{~GB}$, and $80 \mathrm{~GB}$, respectively.

For the sake of accuracy, each experiment has been repeated for 30 times, and the performance and the energy consumption results reported in this subsection are the average numbers of all repetitions. We ran the three targeted MapReduce benchmarks on 
Table 3 Different configurations for running Hadoop MapReduce benchmarks

\begin{tabular}{lll}
\hline Experiment number & Application host configuration & $\begin{array}{l}\text { Enabled in-storage } \\
\text { processing } \\
\text { capability }\end{array}$ \\
\hline 1 & Low & None \\
2 & Low & 2 ISP-enabled CSDs \\
3 & Low & 4 ISP-enabled CSDs \\
4 & Low & 6 ISP-enabled CSDs \\
5 & Medium & None \\
6 & Medium & 2 ISP-enabled CSDs \\
7 & Medium & 4 ISP-enabled CSDs \\
8 & Medium & 6 ISP-enabled CSDs \\
9 & High & None \\
10 & High & 2 ISP-enabled CSDs \\
11 & High & 4 ISP-enabled CSDs \\
12 & High & 6 ISP-enabled CSDs \\
13 & High & 8 ISP-enabled CSDs \\
14 & High & 10 ISP-enabled CSDs \\
15 & High & 12 ISP-enabled CSDs \\
16 & High & 16 ISP-enabled CSDs \\
\hline
\end{tabular}

the 16 different platform configurations, and each experiment has been repeated 30 times that gives us a total of 1440 MapReduce tests.

As previously stated, in all experiments, the application host uses all connected Catalina CSDs as storage units, however, in each test, a certain number of CSDs are enabled to run MapReduce application in-place and play the role of a processing node. Figures 10, 11 shows the Hadoop MapReduce experiments performance and energy consumption results, respectively.

The diagrams in Fig. 10 show that increasing the number of ISP-enabled CSDs decreases the elapsed time for all benchmarks. The performance of the high-configured application host platform increased up to $2.2 \times$ when the ISP engines of all 16 Catalina CSDs are enabled. Thus, deploying ISP-enabled CSDs increases the performance of the Hadoop MapReduce benchmarks significantly.

Also, according to these diagrams, the elapsed time for running the MapReduce benchmarks on the low-configured application host platform equipped with six Catalina CSDs is close to the elapsed time of running the benchmarks on the high-configured application host platform with no enabled ISP engine. So, only six Catalina CSDs can improve the performance of a low-end host close to the performance of a high-end host. As we previously discussed, the cost of implementing the ISP engine inside the SSDs is negligible compared to the total cost of manufacturing an SSD, so ISP technology can considerably improve the performance of Hadoop clusters economically. Figure 11 shows the energy consumption results of running the Hadoop MapReduce benchmarks on the developed platform for different configurations.

According to Fig. 11, the energy consumption of running the benchmarks on the lowconfigured application host platform decreases up to 36\% by deploying six ISP-enabled Catalina CSDs. This improvement for the high-configured application host platform equipped with 16 ISP-enabled Catalina CSDs reaches to $4.3 \times$. 


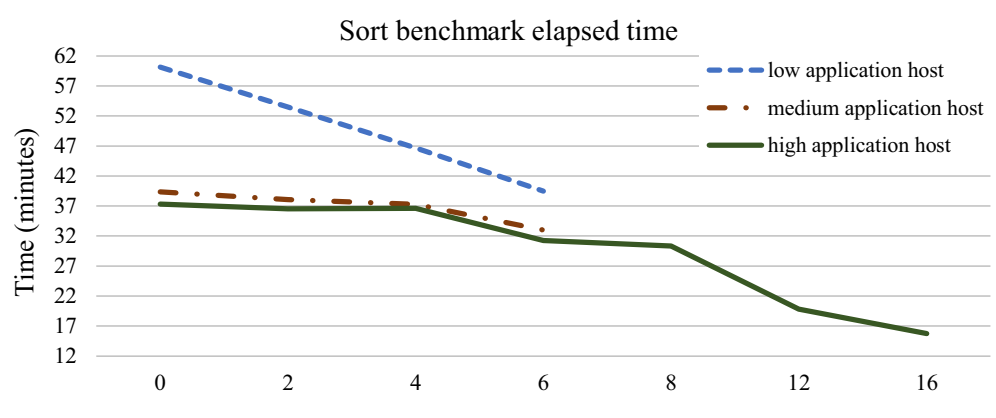

Terasort benchmark elapsed time

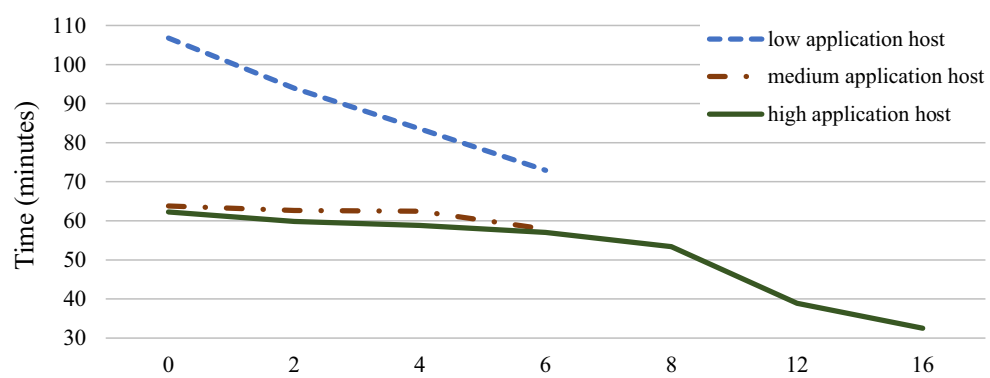

Wordcount benchmark elapsed time

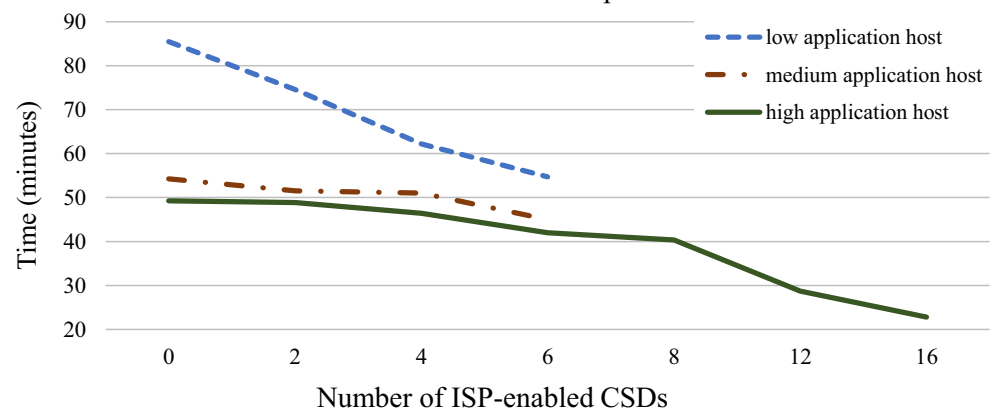

Fig. 10 Hadoop MapReduce benchmarks performance results

With no ISP engine enabled, the low-configured application host platform is less energy efficient than the other configurations. This is expected behavior since running the same benchmark on a more powerful platform takes less time. According to the diagrams in Fig. 11, when we enabled six ISP engines, the energy efficiency of the low-configured application host platform can surpass the energy efficiency of the medium-and high-configured application host platforms equipped with the same number of ISP-enabled CSDs. Although, the performance of the low-configured application host platform is still lower than the other platforms (see Fig. 10). We believe that this happens because of the high energy efficiency of the Catalina CSDs.

The Hadoop framework distributes tasks among all of the processing nodes. If a processing node gets idle, it will fetch data from other busy nodes and process it. Thus, the amount of data processed by each node in the Hadoop cluster is proportional to its processing resources. This means in the low-configured application host platform, a larger amount of data is processed by the Catalina CSDs compared to the amount of data processed by them in the high-configured application host platform which has an Intel Xeon processor. Since 

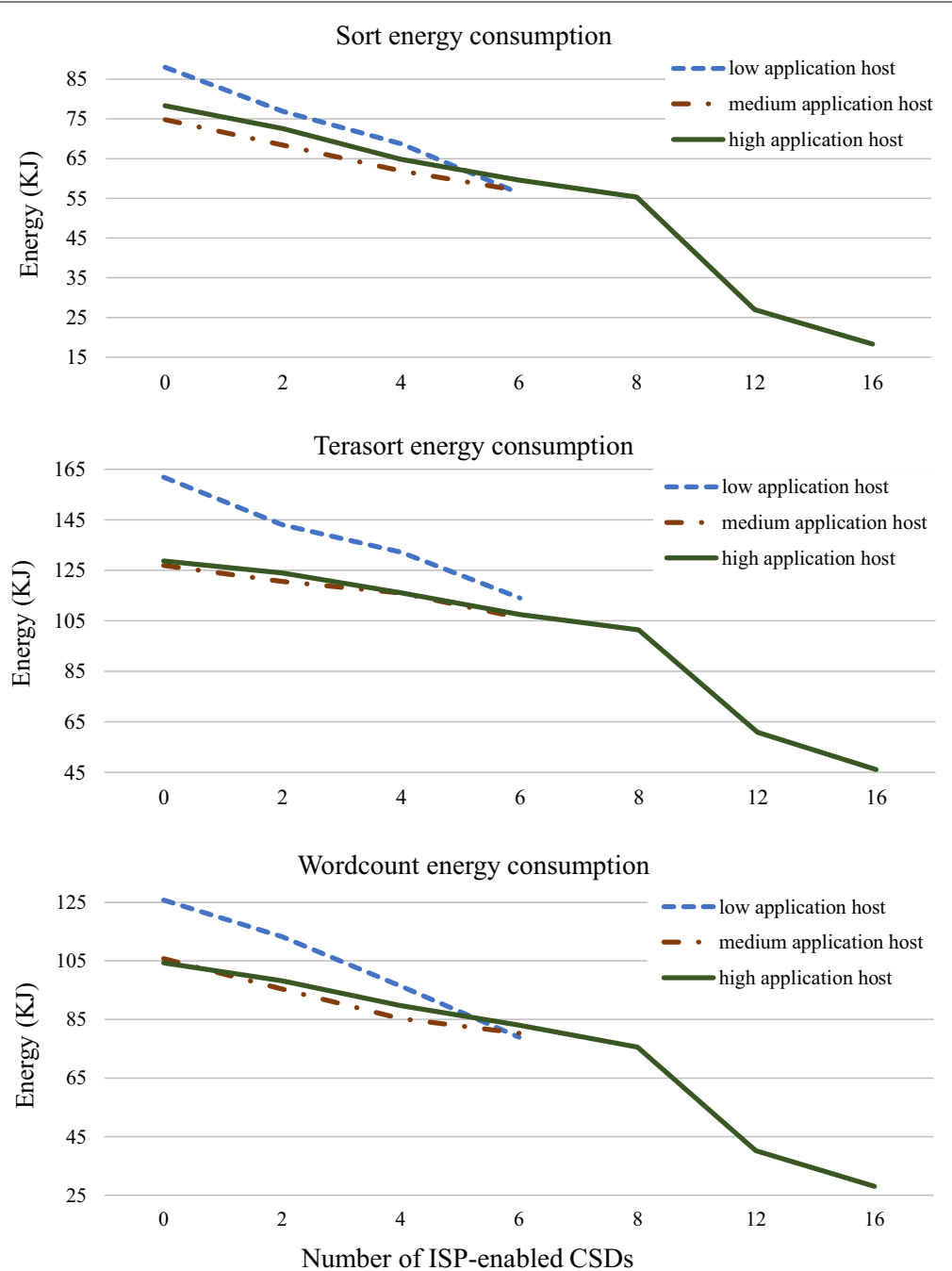

Fig. 11 Hadoop MapReduce benchmarks energy consumption results

ISP engines are considerably more energy-efficient than the application host's processor, as we increase the portion of data processed by the CSDs, the whole platform becomes more energy-efficient. This justifies why the energy efficiency of the low-configured application host platform equipped with 6 ISP-enabled CSDs can surpass the energy efficiency of the high-configured application host platform with the same number of ISP-enabled Catalina CSDs.

\section{HPC benchmarks and results}

In this subsection, we first describe the targeted benchmarks to investigate the effect of deploying Catalina CSDs in clusters for running HPC applications. Then we show and discuss the performance and energy consumption results of running the benchmarks on the developed platform. The HPC applications usually demand a considerable amount 
of processing resources and consume a large amount of data. Thus, we only consider the high-configured application host platform equipped with 16 Catalina CSDs for running the HPC experiments (see Table 2). We have implemented the MPI framework to run the HPC benchmark according to the architecture described earlier in this section. The MPI coordinator runs on the head node host, while the application host and the ISP-enabled Catalina CSDs, run the MPI workers. In the developed platform, the application host can access the data stored on all the Catalina CSDs; however, each CSD only has access to its local data.

In addition, for running the HPC applications in-place, Catalina CSDs should be able to deliver a compelling performance. Therefore, we have utilized the Neon SIMD engines inside the Catalina CSDs. The Neon SIMD engines are application-specific integrated circuit (ASIC) accelerators that are expected to improve the performance and energy efficiency of the applications significantly. Overall, this section shows how using ASIC-based accelerators enhances the benefits of deploying CSDs for running HPC applications.

The HPC Challenge benchmark suite [32] which is developed by the University of Tennessee is one of the well-known HPC benchmark suites and is used in many research works [33-35]. This suite is composed of several benchmarks, each of which focuses on a particular feature of the HPC clusters such as the ability to do floating-point calculations, the communication speed between nodes, and the potentials of running demanding algorithms such as DFT. Among these benchmarks, we have targeted the DFT algorithm, since it is a CPU-intensive algorithm that also consumes a large amount of data, so it can show the potentials of deploying CSDs in clusters. Also, DFT is one of the most important algorithms, as Gilbert Strang, the author of the textbook Linear Algebra and Its Applications [36] referred to it as "the most important numerical algorithm in our lifetime." The DFT of a finite sequence $X$ is a finite sequence $Y$ with the same length of $X$ in a complex-valued format in the frequency domain. The DFT of the finite sequence $X$ is defined by (1).

$$
\begin{aligned}
Y & =F\left\{x_{n}\right\} \\
y_{k} & =\sum_{n=0}^{N-1} x_{n} \cdot e^{-\frac{2 \pi i}{N} k n}
\end{aligned}
$$

In the case of multidimensional input signal of $X:\left\{x_{n_{1}, n_{2}, \ldots, n_{l}}\right\}$, a d-dimensional DFT is defined as (2).

$$
\begin{aligned}
y_{k_{1}, k_{2}, \ldots, k_{1}} & =\sum_{n_{1}=0}^{N_{1}-1}\left(\alpha_{N_{1}}^{n_{1} k_{1}} \sum_{n_{2}=0}^{N_{2}-1}\left(\alpha_{N_{2}}^{n_{2} k_{2}} \ldots \sum_{n_{d}=0}^{N_{d}-1}\left(\alpha_{N_{d}}^{n_{d} k_{d}} \cdot x_{n_{1}, n_{2}, \ldots, n_{d}}\right)\right)\right) \\
\text { where } \alpha_{N_{l}} & =\exp \left(\frac{-2 \pi}{N_{l}}\right)
\end{aligned}
$$

Considering a large amount of floating-point input data, the multi-dimensional DFT calculation is a challenging CPU-intensive application and can show the potentials of the Catalina CSDs for running HPC applications. Thus, we targeted this algorithm to measure the energy consumption and performance of 1D, 2D, and 3D DFT calculations of large datasets running on the high-configured application host platform with different number of ISP-enabled Catalina CSDs. For implementing the DFT algorithm, we utilized the FFTW library [37], which can be compiled to use the Neon SIMD engines of 
Catalina CSDs, and also supports multi-threading capability of the processing nodes in the developed platform.

For running the 1D-, 2D-, and 3D-DFT calculations, we have prepared three different datasets. The PTB Diagnostic ECG dataset is used for 1D-DFT calculation. The PTB Diagnostic ECG is a set of ECG signals collected from healthy volunteers and patients with different heart diseases by Professor Michael Oeff, M.D., at the Department of Cardiology of University Clinic Benjamin Franklin in Berlin, Germany [38, 39]. We have duplicated this dataset to generate 200 million $1 \mathrm{D}$ objects, each of which is a sequence of 180 floating-point numbers.

Regularly, 2D-DFT operations are performed on images; therefore, we have generated 14.4 million synthetic grayscale images as the 2D-DFT dataset. On each of these images, a dark point is placed randomly on the image, and other points' brightness is relative to their distance from the single darkest point. Figure 12 shows four samples of these images. For performing 2D-DFT operations, we converted each of the images to a $50 \times 50$ matrix. Overall, the 2D-DFT dataset is composed of 14.4 million $2 \mathrm{D}$ objects, where each of which is a sequence of 2500 floating-point numbers.

The 3D dataset also is generated using the same method we used for generating the 2D dataset. Each object in the 3D dataset can be described as a cube-shaped 3D object where a single darkest point is placed randomly in the cube-shaped object, and other points' brightness is relative to their distance from the single darkest point. We have generated a set of 288,000 three-dimensional objects and converted them to $50 \times 50 \times 50$ matrices to represent the dataset for the 3D-DFT operations. Table 4 summarizes the datasets we used for running 1D-, 2D-, and 3D-DFT operations on the developed CSDenabled platform.


Fig. 12 Four samples of the synthetic images used for 2D-DFT calculations 
Similar to the Hadoop MapReduce experiments, in all of the DFT calculation experiments, the application host has access to the data stored in all of the Catalina CSDs and the CSDs always play the role of storage units. However, in each test, a certain number of ISP-engines of the CSDs are enabled to show the scalability of ISP technology for running HPC applications. Figure 13 shows the performance and energy consumption results of running the DFT calculations on the developed platform for different numbers of ISP-enabled Catalina CSDs. The performance reported in the diagrams is defined as the number of $1 \mathrm{D}, 2 \mathrm{D}$, and $3 \mathrm{D}$ objects that have been processed in a second, and the reported energy consumption is the energy consumed for processing an object. It is worth mentioning that each test has been repeated 20 times, and each result reported in this subsection is the average of all repetitions.

Table 4 Datasets for 1D-, 2D-, and 3D-DFT calculations

\begin{tabular}{llll}
\hline Dataset & Number of objects & Dimensions of an object & $\begin{array}{l}\text { Total size } \\
\text { of the dataset } \\
\text { (GB) }\end{array}$ \\
\hline 1D-DFT & 200 million & $180 \times 1$ & 288 \\
2D-DFT & 14.4 million & $50 \times 50$ & 288 \\
3D-DFT & 288,000 & $50 \times 50 \times 50$ & 288 \\
\hline
\end{tabular}

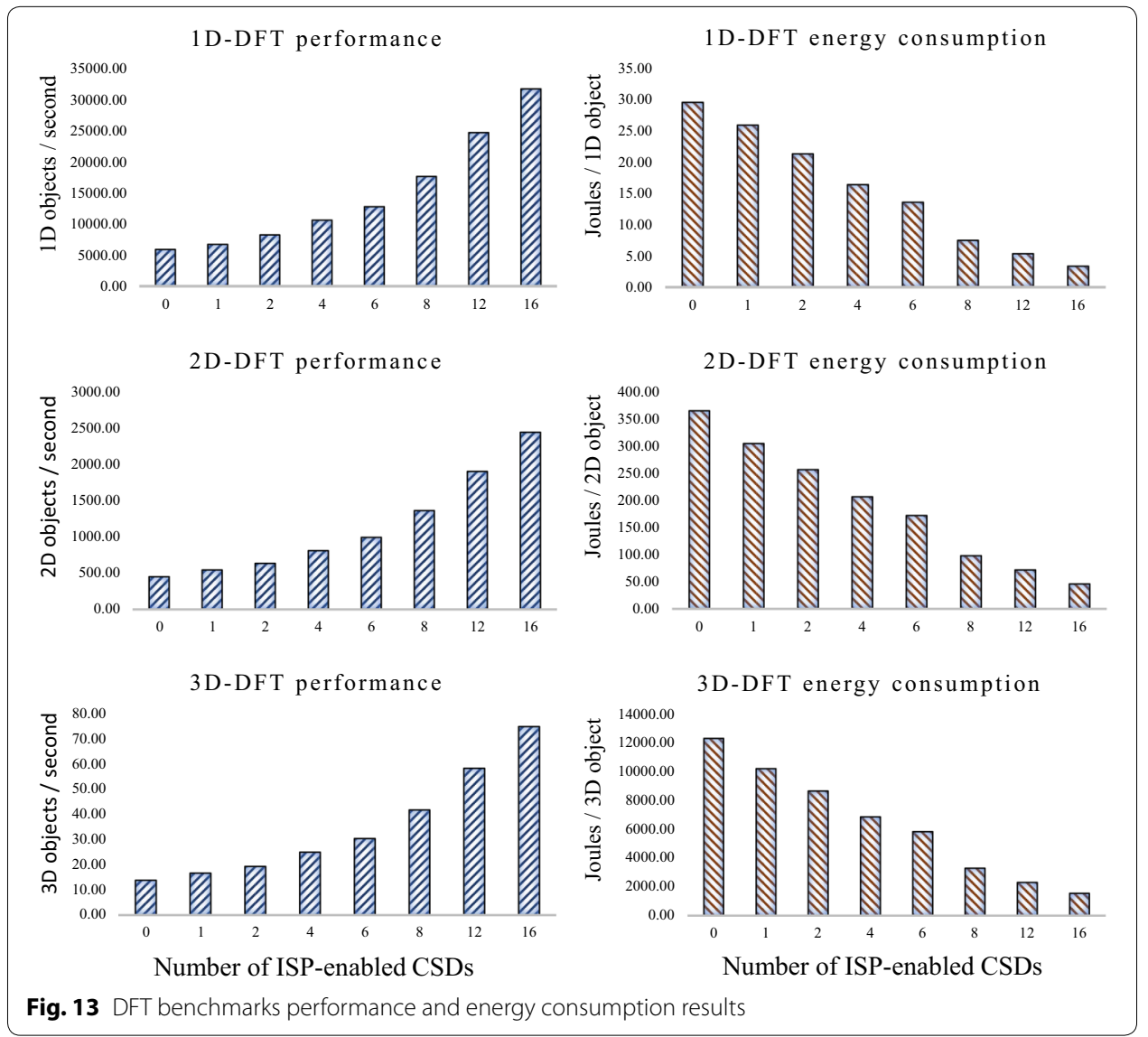


According to the diagrams in Fig. 13, as we enable more ISP-engines, the performance increases, and the energy consumption decreases. In these experiments, adding 16 ISP-enabled Catalina CSDs has improved the performance and energy consumption of running DFT calculations by a factor of $5.4 \times$ and $8.9 \times$, respectively. The comparison between the results of running the Hadoop MapReduce and the HPC benchmarks yields an important outcome. The deployment of Catalina CSD in the platform improves the performance and energy consumption of running DFT calculations significantly more than the Hadoop MapReduce benchmarks. We believe that this difference is rooted in the utilization of Neon SIMD engines for running the DFT calculations. In other words, the Neon SIMD engines accelerate the execution of the DFT algorithm considerably. Since in Catalina CSDs, these engines are close to where data reside, they make a compelling improvement when ISP engines utilize them for running the applications in-place.

\section{Related works}

With the emergence of SSDs, the gap between the internal bandwidth of the storage units and the bandwidth of common I/O interfaces such as SATA, SAS, and NVMe has increased significantly up to an order of magnitude. This gap, along with the considerable power usage in nowadays data centers, calls for "moving the process closer to data" [40]. Early studies show that omitting low-speed I/O interfaces can give considerable speedup to data-intensive applications and lower the overall energy consumption [28]. Such studies bold the importance of near data processing.

The ISP technology is a relatively new trend in the field of near data processing. This technology enables the storage units to run user applications in-place without sending the data to a host. Since integrating a dedicated general-purpose processing engine inside modern SSD architecture is time-consuming and comes with considerable design challenges, most ISP works either use the native processing engine inside SSDs that is originally intended to run conventional flash management routines or embed an FPGAbased accelerator inside the SSD to run user application in-place.

Thus, we have categorized some of the related ISP works into the following groups: ISP works that use native SSD's processor to run user commands $[29,41]$ and ISP projects that bundle an auxiliary processing engine (usually FPGA) [24, 42-44]. Biscuit [42] is a framework to run applications in a distributed fashion among one host and several SSD units. The framework proposes a flow-based programming model for developing ISP applications. It decomposes the developed applications into small code sections, called SSDlet, and dynamically distributes the SSDlets among the SSDs. One of the drawbacks of Biscuit framework is that there is no dedicated processing engine for ISP and it uses the same ARM cortex R7 real-time processor that is intended to run conventional SSD firmware as the ISP engine. This method can greatly impact the host's interface performance, which makes it unsuitable for host systems with lots of I/O interactions. Also, Biscuit requires applications to be developed using a flow-based programming model. This remarkably limits the reuse of other types of applications. The research works [29, 41] have the same approach of using a processing engine both for conventional SSD and ISP routines; however, the scope of these works are limited to Hadoop mapper tasks and SQL scan and join, respectively. 
BlueDBM proposes a pure FPGA architecture using Virtex7 FPGA, which has enough resources both for SSD controller and in-storage processing [43]. In BlueDBM, storage units contain a network interface implemented in FPGA to form a network for running user applications in a distributed fashion. The most notable drawback of an ISP-enabled storage architecture using pure FPGA implementation is the time-consuming design process due to its reconfigurability issues. In other words, users have to go through the entire process of redesigning, synthesizing, place, and route, to generating a bitstream for implementing the desired ISP functionality [45]. Jo et al. [46], proposes a heterogeneous platform composed of CPU, GPU and ISP-enabled SSD (called iSSD). Based on simulations, this platform can get up to $3.5 \times$ speedup for data-intensive algorithms. Although this method looks effective, it never went beyond simulation. In all the mentioned related works, lack of an OS inside ISP-enabled storage makes it harder to adopt applications for running in-place.

\section{Conclusion}

The ISP technology enables storage units to run user applications in-place, i.e., data is not required to move from the storage units to the host's memory to be processed. It can relieve the data movement challenges in the big data applications when huge data need to be fetched from the storage systems. The modern solid-state drives (SSDs) provide a better environment for implementing ISP technology in comparison to the traditional hard disk drives (HDDs). The computational storage devices (CSDs) are storage units based on the modern SSD architecture, which can run user applications in-place.

In this paper, we introduce Catalina, an efficient and flexible computational storage platform featuring a dedicated ISP engine including a quad-core ARM application processor, as well as Neon SIMD engines that can be utilized for accelerating HPC applications that run in-place. Catalina is equipped with a full-fledged Linux OS which provides a flexible environment for running a vast spectrum of applications. The applications running inside Catalina has filesystem-level access to the data stored in flash memory. Also, a TCP/IP tunnel through NVMe/PCIe link has been developed that allows Catalina to communicate with the host. Catalina can be seamlessly deployed in distributed environments such as Hadoop and MPI-based clusters. For the proof of concept, we have built a fully functional Catalina prototype as well as a system equipped with 16 Catalina CSDs to investigate the benefits of deploying the CSDs in clusters. The experimental results show that the deployment of Catalina in the clusters improves the MapReduce application performance and the power efficiency up to $2.2 \times$ and $4.3 \times$, respectively. By utilizing the Neon SIMD engines for accelerating DFT algorithms, the performance and power efficiency improvements grow even higher up to $5.4 \times$ and $8.9 \times$, respectively.

\footnotetext{
Abbreviations

API: application programming interfaces; ASIC: application specific integrated circuit; AXI: Xilinx advanced extensible interface; CSD: computational storage device; DFT: discrete Fourier transform; ECG: electrocardiogram; FPU: floatingpoint units; FTL: flash translation layer; GPU: graphics processing unit; HDD: hard disk drive; HDFS: Hadoop filesystem; HPC: high-performance computing; ISP: in-storage processing; MPI: message passing interface; MPSoC: multi-processor system-on-chip; NFS: network filesystem; NVMe: non-volatile memory express; OCFS: Oracle cluster filesystem; OS: operating system; PCle: peripheral component interconnect express; PL: programmable logic; PS: processing system; SIMD: single instruction, multiple data; SSD: solid-state drive.
} 


\section{Acknowledgements}

Not applicable.

\section{Authors' contributions}

The author MT prepared the draft of all sections except the "Related work" section and also ran the experiments and collected the results. AH prepared the draft for "Related work" section. The authors MT, SR, AH, and HB participated in developing the proposed solution and also helped to prepare the final manuscript. All authors read and approved the final manuscript.

\section{Funding}

This work was supported by the USA National Science Foundation under Award number 1660071.

\section{Availability of data and materials}

Not applicable.

\section{Competing interests}

The authors declare that they have no competing interests.

\section{Author details}

${ }^{1}$ University of California, Irvine (UCI), Irvine 92697, USA. ${ }^{2}$ NGD Systems, Inc., 355 Goddard, Suite 200, Irvine 92618, USA.

Received: 3 August 2019 Accepted: 4 November 2019

Published online: 15 November 2019

\section{References}

1. James J. Data never sleeps 6.0. 2018. https://www.domo.com/blog/data-never-sleeps-6. Accessed 11 Nov 2019.

2. Javani A, Zorgui M, Wang Z. Age of information in multiple sensing. 2019. arXiv:1902.01975.

3. Kitchin R, McArdle G. What makes big data, big data? exploring the ontological characteristics of 26 datasets. Big Data Soc. 2016:3:1-10.

4. Pfister GF. An introduction to the infiniband architecture. In: High performance mass storage and parallel I/O. 2001:42:617-32.

5. Boden NJ, Cohen D, Felderman RE, Kulawik AE, Seitz CL, Seizovic JN, Su W-K. Myrinet: a gigabit-per-second local area network. IEEE Micro. 1995;15(1):29-36.

6. Prabhat, Koziol Q. High performance parallel I/O. Cleveland: CRC Press; 2014.

7. Shvachko K, Kuang H, Radia S, Chansler R, et al. The hadoop distributed file system. MSST. 2010;10:1-10.

8. Elyasi N, Choi C, Sivasubramaniam A. Large-scale graph processing on emerging storage devices. In: Proceedings of the 17th USENIX conference on file and storage technologies. FAST'19. Berkeley: USENIX Association; 2019. pp. 309-16.

9. SATA ecosystem web page. https://sata-io.org. Accessed 11 Nov 2019.

10. Serial-attached SCSI (SAS) Web page. https://searchstorage.techtarget.com/definition/serial-attached-SCSI. Accessed 11 Nov 2019.

11. Benefits of using NVMe over PCI Express Fabrics. https://www.dolphinics.com/solutions/nvme_over_pcie_fabri cs.html. Accessed 11 Nov 2019.

12. Zynq UltraScale+MPSoC product web page. https://www.xilinx.com/products/silicon-devices/soc/zynq-ultrascale -mpsoc.html. Accessed 11 Nov 2019.

13. Torabzadehkashi M, Rezaei S, HeydariGorji A, Bobarshad H, Alves V, Bagherzadeh N. Catalina: in-storage processing acceleration for scalable big data analytics. In: 2019 27th Euromicro international conference on parallel, distributed and network-based processing (PDP). IEEE. 2019. p. 430-7.

14. Park S, Kim Y, Urgaonkar B, Lee J, Seo E. A comprehensive study of energy efficiency and performance of flash-based ssd. J Syst Archit. 2011;57(4):354-65.

15. The Open Compute Project (OCP) web page. https://www.opencompute.org. Accessed 11 Nov 2019.

16. Seagate Kinetic HDD produce web page. https://www.seagate.com/support/enterprise-servers-storage/nearlinestorage/kinetic-hdd/. Accessed 11 Nov 2019.

17. Patel AB, Birla M, Nair U. Addressing big data problem using hadoop and map reduce. In: 2012 Nirma University International conference on engineering (NUiCONE). IEEE. 2012. p. 1-5.

18. Mashayekhy L, Nejad MM, Grosu D, Zhang Q, Shi W. Energy-aware scheduling of mapreduce jobs for big data applications. IEEE Trans Parallel Distrib Syst. 2014;26(10):2720-33.

19. Vavilapalli VK, Murthy AC, Douglas C, Agarwal S, Konar M, Evans R, Graves T, Lowe J, Shah H, Seth S, et al. Apache hadoop yarn: yet another resource negotiator. In: Proceedings of the 4th annual symposium on cloud computing. ACM. 2013. p. 5

20. Mishra P, Mishra M, Somani AK. Bulk i/o storage management for big data applications. In: 2016 IEEE 24th international symposium on modeling, analysis and simulation of computer and telecommunication systems (MASCOTS). IEEE. 2016. p. 412-7.

21. Mishra P, Somani AK. LDM: lineage-aware data management in multi-tier storage systems. In: Future of information and communication conference. Springer. 2019. p. 683-707.

22. White T. Hadoop: the definitive guide. Sebastopol: O'Reilly Media Inc; 2012

23. Kishani M, Tahoori M, Asadi H. Dependability analysis of data storage systems in presence of soft errors. IEEE Trans Reliab. 2019:68:201-15. 
24. Torabzadehkashi M, Rezaei S, Alves V, Bagherzadeh N. Compstor: an in-storage computation platform for scalable distributed processing. In: 2018 IEEE international parallel and distributed processing symposium workshops (IPDPSW). IEEE. 2018. p. 1260-7.

25. Torabzadehkashi M, HeydariGorji A, Rezaei S, Bobarshad H, Alves V, Bagherzadeh N. Accelerating HPC applications using computational storage devices. IEEE. 2019. p. 1878-85.

26. Walker DW, Dongarra JJ. MPI: a standard message passing interface. Supercomputer. 1996;12:56-68.

27. Oracle cluster filesystem second version web page. https://oss.oracle.com/projects/ocfs2/. Accessed 11 Nov 2019.

28. Cho S, Park C, Oh H, Kim S, Yi Y, Ganger GR. Active disk meets flash: a case for intelligent SSDs. In: Proceedings of the 27th international ACM conference on international conference on supercomputing. ICS '13. 2013. p. 91-102.

29. Kim S, Oh H, Park C, Cho S, Lee S-W, Moon B. In-storage processing of database scans and joins. Inf Sci. 2016;327:183-200.

30. The CUBIX XPANDER produce web page. https://www.cubix.com/xpander/. Accessed 11 Nov 2019.

31. Huang S, Huang J, Dai J, Xie T, Huang B. The hibench benchmark suite: characterization of the mapreduce-based data analysis. In: 2010 IEEE 26th international conference on data engineering workshops (ICDEW 2010). IEEE. 2010. p. 41-51.

32. The HPC Challenge benchmark suite web page. http://www.hpcchallenge.org. Accessed 11 Nov 2019.

33. Dongarra J, Heroux MA. Toward a new metric for ranking high performance computing systems. Sandia Report, SAND2013-4744 312, 150; 2013.

34. Dongarra J, Luszczek P. Reducing the time to tune parallel dense linear algebra routines with partial execution and performance modelling. University of Tennessee Computer Science Technical Report, Tech. Rep. 2010

35. Steinbach P, Werner M. gearshifft-the FFT benchmark suite for heterogeneous platforms. In: International supercomputing conference. Springer. 2017. p. 199-216.

36. Rose NJ. Linear algebra and its applications (gilbert strang). SIAM Rev. 1982;24(4):499-501.

37. Frigo M, Johnson SG. The design and implementation of FFTW3. Proc IEEE. 2005;93(2):216-31.

38. Bousseljot R, Kreiseler D, Schnabel A. Nutzung der ekg-signaldatenbank cardiodat der ptb über das internet. Biomed Eng. 1995;40(s1):317-8.

39. Goldberger AL, Amaral LA, Glass L, Hausdorff JM, Ivanov PC, Mark RG, Mietus JE, Moody GB, Peng C-K, Stanley HE. Physiobank, physiotoolkit, and physionet: components of a new research resource for complex physiologic signals. Circulation. 2000;101(23):215-20.

40. Do J, Kee Y-S, Patel JM, Park C, Park K, DeWitt DJ. Query processing on smart SSDs: opportunities and challenges. In: Proceedings of the 2013 ACM SIGMOD international conference on management of data. SIGMOD '13. 2013. p. 1221-30.

41. Park D, Wang J, Kee Y. In-storage computing for hadoop mapreduce framework: challenges and possibilities. IEEE Trans Comput. 2018; . https://doi.org/10.1109/TC.2016.2595566.

42. Gu B, Yoon AS, Bae D, Jo I, Lee J, Yoon J, Kang J, Kwon M, Yoon C, Cho S, Jeong J, Chang D. Biscuit: a framework for near-data processing of big data workloads. In: 2016 ACM/IEEE 43rd annual international symposium on computer architecture (ISCA). 2016. p. 153-165.

43. Jun S-W, Liu M, Lee S, Hicks J, Ankcorn J, King M, Xu S, Arvind. BlueDBM: an appliance for big data analytics. SIGARCH Comput Archit News. 2015;43(3):1-13.

44. Song $X$, Xie T, Pan W. RISP: a reconfigurable in-storage processing framework with energy-awareness. In: 2018 18th IEEE/ACM international symposium on cluster, cloud and grid computing (CCGRID). 2018. p. 193-202.

45. Rezaei S, Kim K, Bozorgzadeh E. Scalable multi-queue data transfer scheme for FPGA-based multi-accelerators. In: 2018 IEEE 36th international conference on computer design (ICCD). IEEE. 2018. p. 374-80.

46. Jo Y-Y, Cho S, Kim S-W, Oh H. Collaborative processing of data-intensive algorithms with CPU, intelligent SSD, and GPU. In: Proceedings of the 31st annual ACM symposium on applied computing. SAC '16. New York: ACM. 2016. p. 1865-70.

\section{Publisher's Note}

Springer Nature remains neutral with regard to jurisdictional claims in published maps and institutional affiliations.

\section{Submit your manuscript to a SpringerOpen ${ }^{\circ}$ journal and benefit from:}

- Convenient online submission

- Rigorous peer review

- Open access: articles freely available online

- High visibility within the field

- Retaining the copyright to your article

Submit your next manuscript at $\boldsymbol{\Delta}$ springeropen.com 